\title{
O impacto da estratégia saúde da família sobre os indicadores da atenção básica no Brasil
}

\author{
The impact of the family health strategy on the primary care health indicators in Brazil \\ El impacto de la estrategia familiar de salud en los indicadores de atención básica en \\ Brasil
}

Amanda Fróes Ribeiro ${ }^{1 *}$, Gabriel Felipe Albuquerque Barbosa ${ }^{1}$, Thayná Marques Alves ${ }^{1}$, Ítalo Filipe França $^{1}$, Andreza Martins de Lima ${ }^{1}$, Marinilza Soares Mota Sales ${ }^{1}$, Cássia Pérola dos Anjos Braga Pires ${ }^{1}$, Gislaine Conceição Teixeira Pereira e Maia ${ }^{1}$, Renata Francine Rodrigues de Oliveira ${ }^{1}$, Aline Soares Figueiredo Santos ${ }^{1}$.

\section{RESUMO}

Objetivo: Analisar o impacto da Estratégia Saúde da Família (ESF) sobre os indicadores de saúde da atenção básica no Brasil. Métodos: Realizou-se uma revisão integrativa com a questão central: Qual o impacto da Estratégia Saúde da Família nos indicadores da Atenção Básica no Brasil? Definiram-se os descritores por meio das plataformas Descritores em Ciências da Saúde (DeCS) e Medical Subject Headings (MeSH), os quais foram utilizados para a busca dos estudos, finalizando a amostra dessa revisão com o total de 07 artigos. Resultados: Os resultados foram analisados e discutidos, tendo predominado abordagens sobre impacto da ESF nos indicadores de saúde na atenção básica, internações por condições sensíveis à atenção primária e resultados alcançados com a Estratégia Saúde da Família. Outros temas também se destacaram, como mortalidade infantil, controle da tuberculose na ESF e doenças infecciosas e parasitárias. Considerações finais: Percebeu-se que houve uma melhoria significativa nos indicadores de saúde apresentados, com o estabelecimento da ESF na atenção básica do Brasil, acarretando em diferentes vantagens para a qualidade de vida da população investigada, bem como para o serviço de saúde pública do Brasil.

Palavras-chave: Estratégia saúde da família, Indicadores básicos de saúde, Atendimento básico.

\begin{abstract}
Objective: Analyze the impact of the Family Health Strategy (FHS) on primary health care indicators in Brazil. Methods: An integrative review was carried out with the central question: What is the impact of the Family Health Strategy on the Primary Care indicators of Brazilians? Descriptors were defined using the Health Sciences Descriptors (DeCS) and Medical Subject Headings (MeSH) platforms, which were used to search for studies, ending the sample of this review with a total of 07 articles. Results: The results were analyzed and discussed, with approaches on the impact of the FHS on health indicators in primary care, hospitalizations due to conditions sensitive to primary care, and results achieved with the Family Health Strategy predominating. Other themes also stood out, such as infant mortality, tuberculosis control in the FHS and infectious and parasitic diseases. Final considerations: It was noticed that there was a significant improvement in the health indicators presented, with the establishment of the FHS in primary care in Brazil, resulting in different advantages for the quality of life of the investigated population, as well as for the public health service in Brazil.
\end{abstract}

Keywords: Family health strategy, Basic health indicators, Basic care.

${ }^{1}$ Universidade Estadual de Montes Claros (UNIMONTES), Montes Claros - MG.

*E-mail: froesribeiro10@gmail.com

SUBMETIDO EM: 7/2020

I ACEITO EM: 8/2020

PUBLICADO EM: 11/2020

REAS/EJCH | Vol.12(11) | e4730 | DOI: https://doi.org/10.25248/reas.e4730.2020

Página 1 de 9 
RESUMEN

Objetivo: Analizar el impacto de la Estrategia de Salud Familiar (ESF) en los indicadores de atención primaria de salud en Brasil. Métodos: Se realizó una revisión integradora con la pregunta central: ¿Cuál es el impacto de la Estrategia de Salud Familiar en los indicadores de Atención Primaria de los brasileños? Los descriptores se definieron mediante las plataformas Descriptores en Ciencias de la Salud (DeCS) y Medical Subject Headings (MeSH), que se utilizaron para buscar estudios, terminando la muestra de esta revisión con un total de 07 artículos. Resultados: Los resultados fueron analizados y discutidos, con enfoques sobre el impacto del ESF en los indicadores de salud en la atención primaria, hospitalizaciones debido a condiciones sensibles a la atención primaria y los resultados logrados con la estrategia de salud familiar predominante. También se destacaron otros temas, como la mortalidad infantil, el control de la tuberculosis en la ESF y las enfermedades infecciosas y parasitarias. Consideraciones finales: Se observó que hubo una mejora significativa en los indicadores de salud presentados, con el establecimiento de la ESF en atención primaria en Brasil, lo que resultó en diferentes ventajas para la calidad de vida de la población investigada, así como para el servicio de salud pública en Brasil.

Palabras clave: Estrategia de salud familiar, Indicadores básicos de salud, Atención básica.

\section{INTRODUÇÃO}

De acordo com o relatório final da $8^{\circ}$ Conferência Nacional de Saúde de 1986, direito à saúde significa garantia, pelo Estado, de condições dignas de vida e acesso universal e igualitário às ações e serviços de promoção, proteção e recuperação da saúde levando em consideração o desenvolvimento pleno do ser humano em sua individualidade (BRASIL, 1986). Diante disso, foi aprovada uma proposta que transformou a saúde pública do Brasil, por meio da reestruturação do sistema nacional de saúde que resultou na criação do Sistema Único de Saúde (SUS), a partir da Constituição Federal de 1988, nos artigos 196 ao 200, que afirmam que compete ao SUS: controlar e fiscalizar procedimentos, produtos e substâncias de interesse para a saúde e participar da produção de medicamentos, equipamentos, imunobiológicos, hemoderivados e outros insumos (BRASIL, 1988).

Além disso, também concerne ao SUS executar ações de vigilância sanitária e epidemiológica, ordenar a formação de recursos humanos na área da saúde, participar da formulação política e da execução de ações de saneamento básico, fiscalizar e inspecionar alimentos, incrementar em sua área de atuação o desenvolvimento científico e tecnológico, participar do controle e fiscalização da produção, transporte, guarda e utilização de substâncias e produtos psicoativos, tóxicos e radioativos e colaborar na proteção do meio ambiente (PINTO LF e GIOVANELLA L, 2018). Posteriormente, o SUS foi regulamentado em 1990 pelas Leis 8.080 e 8.142 (BRASIL, 1990a, 1990b).

A Lei 8.080/90 afirma que as ações e serviços públicos de saúde e os serviços privados contratados ou conveniados que integram o SUS são desenvolvidos obedecendo aos princípios de: universalidade de acesso, equidade, integralidade, regionalização e hierarquização, descentralização e comando único e participação popular (BRASIL, 1990a). A Lei 8.142/90 dispõe sobre a participação da comunidade na gestão do SUS e sobre as transferências financeiras intergovernamentais na área da saúde (BRASIL, 1990b). No Art. $1^{\circ} \S 2^{\circ}$ infere-se que o Conselho de Saúde será composto por representantes do governo, prestadores de serviço, profissionais da saúde e usuários, que irão atuar na formulação de estratégias e no controle da execução de políticas de saúde, bem como nos aspectos econômicos e financeiros (BRASIL, 1990b).

Também como legislação estruturante, em 1994 foi criado o Programa Saúde da Família (PSF) com o objetivo de reorganizar a atenção básica que, gradualmente, foi tornando-se a principal estratégia para a mudança do modelo assistencial (PINTO LF e GIOVANELLA L, 2018). No ano de 1997, a portaria 1886 do dia 18 de dezembro, estabelece o PSF como Estratégia Saúde da Família (ESF) (BRASIL, 1997). Nessa modalidade de atenção à saúde, a população passa a ser assistida por uma equipe multiprofissional, composta por um médico, um enfermeiro, um ou dois técnicos de enfermagem e quatro a seis agentes comunitários de saúde (BRASIL, 1997). 
Em 2000, a Equipe de Saúde Bucal passa a ser incluída na ESF, com um cirurgião-dentista, um auxiliar de saúde bucal ou técnico de saúde bucal (BRASIL, 2000). Em 23 de março de 2006, a partir da portaria 648 adveio a primeira Política Nacional de Atenção Básica, que estabelece a ESF como porta de entrada da atenção básica, além disso, é preconizada como ordenadora das Redes de Atenção à Saúde (BRASIL, 2006).

Tal Política foi atualizada em 2011, pela portaria 2488 e em 2017, pela portaria 2436, em que está estabelecida a ESF como estratégia prioritária de atenção à saúde, que visa à reorganização da atenção básica no país de acordo com os princípios e diretrizes do SUS (BRASIL, 2011, 2017).

É considerada também como estratégia de expansão, qualificação e consolidação da Atenção Básica, uma vez que favorece a reorientação do processo de trabalho, com maior potencial de resolutividade e impacto nas condições de saúde individuais e coletivas (BRASIL, 2017).

Todo o processo desse modelo assistencial ou estratégia de atenção precisa ser monitorado e avaliado, por isso é necessário mensurar o impacto da qualidade dos indicadores de saúde de acordo com os programas existentes na Atenção Básica (RONCALLI AG e LIMA KC, 2006).

Os indicadores de saúde são medidas que contêm informações sobre o estado de saúde da população adstrita e o desempenho do sistema de saúde. Convencionou-se classificar os indicadores em seis subgrupos, sendo eles: demográficos, socioeconômicos, mortalidade, morbidade e fatores de risco, recursos e cobertura. O grau de excelência de um indicador consiste em sua validade que é a capacidade de medir o que se pretende e a confiabilidade, que consiste em reproduzir os mesmos resultados quando aplicado em condições similares (RIPSA, 2008).

Considerando a relevância de se monitorar os resultados da ESF e visto que há uma lacuna na literatura no que se refere à abordagem desse tema, o objetivo desta revisão integrativa é analisar o impacto da ESF nos indicadores de saúde da Atenção Básica, de acordo com estudos existentes.

\section{MÉTODOS}

O presente estudo trata-se de uma revisão integrativa da literatura, em que foram reunidos e sintetizados os achados de estudos a respeito do impacto da Estratégia Saúde da Família (ESF) sobre os indicadores da Atenção Básica no Brasil.

Para a identificação do tema e construção da pergunta de pesquisa, foi utilizada a estratégia PCC, do Joana Briggs Institute, que representa um acrônimo para Population, Concept e Context (JBI, 2015). Desta forma definiu-se: P- população do Brasil; C- impacto da Estratégia Saúde da Família e C- indicadores da atenção básica, e a questão norteadora definida foi: Qual o impacto da Estratégia Saúde da Família nos indicadores da atenção básica no Brasil?

A definição dos descritores a serem pesquisados ocorreu conforme busca pelos Descritores em Ciências da Saúde (DeCS) na Biblioteca Virtual em Saúde (BVS) e Medical Subject Headings (MeSH) no National Center for Biotechnology Information (NCBI). Foram definidos 03 grupos de descritores, cada um contendo 03 sinônimos em português e 03 sinônimos em inglês, sendo estes: 1 - Atenção primária à saúde; primeiro nível de atenção à saúde; atendimento básico; care, primary health; health care, primary; primary healthcare; 2 - Indicadores básicos de saúde; indicador de saúde; indicadores do nível de saúde; health status indicator; health status indices; health status index; 3 - Avaliação em saúde; avaliação econômica em saúde; impacto no estado de saúde; healthcare evaluation mechanisms; evaluation mechanism, healthcare; evaluation mechanisms, healthcare.

A coleta de dados ocorreu entre os meses de setembro e outubro de 2019 nas bases de dados Medical Literature Analysis and Retrieval System Online (MEDLINE) e Literatura Latino-Americana e do Caribe em Ciências da Saúde (LILACS), através da plataforma da BVS, na qual houve a combinação dos descritores pré-definidos através do uso dos booleanos OR e AND: tw:((tw:(care, primary health)) OR (tw:(health care, primary)) OR (tw:(primary healthcare)) AND (tw:(health status indicator)) OR (tw:(health status indices)) OR (tw:(health status index)) AND (tw:(healthcare evaluation mechanisms)) OR (tw:(evaluation mechanism, healthcare)) OR (tw:(evaluation mechanisms, healthcare))). 
Os seguintes filtros foram empregados para a pesquisa: texto completo disponível, base de dados MEDLINE e LILACS, o assunto principal foi a Estratégia Saúde da Família, país/região foi o Brasil, idiomas considerados foram português e inglês e o tipo de documento foi artigo.

Os critérios de inclusão adotados consistiram em artigos que avaliassem o impacto da atenção básica longitudinalmente, a partir dos seus indicadores. Os critérios de exclusão foram artigos que apresentassem fuga do tema proposto.

\section{RESULTADOS}

A partir de busca nas bases de dados foram identificados 580 artigos. Inicialmente, realizou-se uma leitura crítica e reflexiva dos títulos e dos resumos encontrados, a partir da qual foi possível estabelecer uma amostra de 76 artigos. Posteriormente, após exclusão dos trabalhos repetidos e leitura dos artigos na íntegra, bem como aplicação dos critérios de inclusão e exclusão, restaram-se 07 artigos para integrar esta revisão. As etapas que compõem o processo de seleção dos estudos estão apresentadas no fluxograma, elaborado por meio da recomendação PRISMA (Principais Itens para Relatar Revisões Sistemáticas e Meta-Análises), que consiste em um conjunto de diretrizes com objetivo de propor avanços, de maneira conceitual e prática, no relato de revisões sistemáticas e meta-análise (Figura 1) (MOHER D, et al., 2015).

Figura 1 - Fluxograma das etapas de análise dos artigos, desenvolvido a partir da recomendação PRISMA.

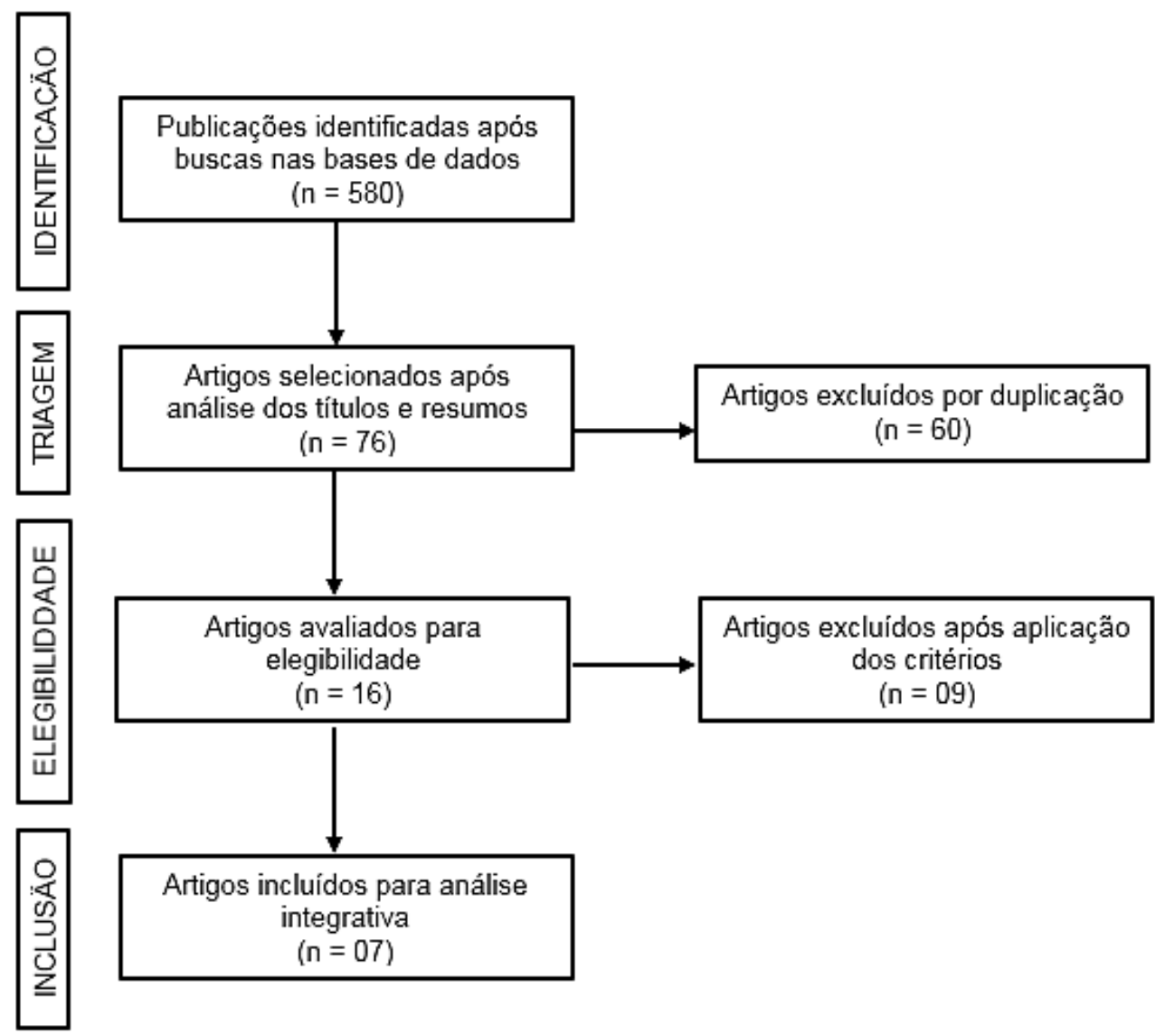

Fonte: Ribeiro AF, et al., 2019.

Considerando os critérios de inclusão e exclusão, os artigos selecionados foram organizados em 2 quadros distintos, o primeiro apresentando autor/ano de publicação, título do artigo, a revista de publicação e o local de realização do estudo (Quadro 1); e o segundo contendo autor/ano de publicação e os principais resultados de cada estudo (Quadro 2). 
Quadro 1 - Descrição dos artigos selecionados quanto à autoria/ ano de publicação, título do artigo, a revista em que foi publicado e o local do estudo.

\begin{tabular}{|c|c|c|c|}
\hline $\begin{array}{l}\text { Autor/ano de } \\
\text { publicacão }\end{array}$ & Título & Revista de publicação & Local de realização do estudo \\
\hline $\begin{array}{l}\text { GUEDES HM, et } \\
\text { al., } 2007\end{array}$ & $\begin{array}{l}\text { Resultados alcançados com a } \\
\text { estratégia saúde da família após cinco } \\
\text { anos de implantação em uma cidade do } \\
\text { interior de Minas Gerais }\end{array}$ & $\begin{array}{l}\text { Revista Mineira de } \\
\text { Enfermagem }\end{array}$ & $\begin{array}{c}\text { Comunidade do Macuco, Município } \\
\text { de Timóteo - MG }\end{array}$ \\
\hline $\begin{array}{l}\text { SANTANA M, et } \\
\text { al., } 2012\end{array}$ & $\begin{array}{l}\text { Efeito da Estratégia Saúde da Família } \\
\text { na vigilância de óbitos infantis }\end{array}$ & Revista Saúde Pública & 417 Municípios da Bahia \\
\hline $\begin{array}{l}\text { MARQUIEVIZ J, } \\
\text { et al., } 2013\end{array}$ & $\begin{array}{l}\text { A Estratégia Saúde da Família no } \\
\text { controle da tuberculose em Curitiba } \\
\text { (PR) }\end{array}$ & $\begin{array}{c}\text { Revista Ciência e Saúde } \\
\text { Coletiva }\end{array}$ & Curitiba - PR \\
\hline $\begin{array}{l}\text { GOMES TGACB, } \\
\text { et al., } 2016\end{array}$ & $\begin{array}{l}\text { Mortalidade na infância no Brasil e } \\
\text { regiões no período de } 2000 \text { a 2011: o } \\
\text { impacto da atenção básica }\end{array}$ & $\begin{array}{l}\text { Revista Comunicação em } \\
\text { Ciências da Saúde }\end{array}$ & Brasil \\
\hline $\begin{array}{l}\text { PAZÓ RG, et al., } \\
2017\end{array}$ & $\begin{array}{l}\text { Panorama das internações por } \\
\text { condições sensíveis à atenção primária } \\
\text { no Espírito Santo, Brasil, } 2000 \text { a } 2014\end{array}$ & $\begin{array}{l}\text { Revista Brasileira de } \\
\text { Medicina de Família e } \\
\text { Comunidade }\end{array}$ & 78 Municípios do Espírito Santo \\
\hline $\begin{array}{l}\text { ZARLOTTI C, et } \\
\text { al., } 2017\end{array}$ & $\begin{array}{l}\text { Internações por condições sensíveis à } \\
\text { atenção primária após implantação da } \\
\text { estratégia saúde da família no } \\
\text { município de Petrópolis/RJ }\end{array}$ & $\begin{array}{l}\text { Revista Online de } \\
\text { Pesquisa: Cuidado é } \\
\text { Fundamental Online }\end{array}$ & Petrópolis - RJ \\
\hline $\begin{array}{l}\text { SILVA-JÚNIOR } \\
\text { VB, et al., } 2018\end{array}$ & $\begin{array}{l}\text { Interface entre as doenças infecciosas e } \\
\text { parasitárias e a Estratégia Saúde da } \\
\text { Família no Brasil }\end{array}$ & $\begin{array}{l}\text { Revista Brasileira de } \\
\text { Ciências da Saúde }\end{array}$ & Brasil \\
\hline
\end{tabular}

Fonte: Ribeiro AF, et al., 2019.

Quadro 2 - Descrição dos artigos selecionados quanto à síntese dos resultados encontrados.

\begin{tabular}{|c|c|}
\hline $\begin{array}{l}\text { Autor/ano de } \\
\text { publicacão }\end{array}$ & Resultados \\
\hline $\begin{array}{l}\text { GUEDES HM, } \\
\text { et al., } 2007\end{array}$ & $\begin{array}{l}\text { Foi possível observar que as visitas domiciliares realizadas pelos membros da equipe, de modo geral, } \\
\text { aumentaram. O número de inalações diminuiu de } 332 \text { para } 202(15,31 \%) \text {, a quantidade de injeções teve uma } \\
\text { queda de } 1.170 \text { para } 355(69,65 \%) \text {, o número de suturas de } 20 \text { para } 4(85,71 \%) \text { e a retirada de pontos de } 32 \\
\text { para } 22(31,25 \% \text {. Esse resultado é considerado positivo, pois indica uma reorientação das práticas de saúde, } \\
\text { nas quais a prevenção e o diagnóstico precoce se mostram eficazes para reduzir as ações curativas. }\end{array}$ \\
\hline $\begin{array}{l}\text { SANTANA M, et } \\
\text { al., } 2012\end{array}$ & $\begin{array}{l}\text { Em } 48,9 \% \text { dos municípios houve investigação de pelo menos um óbito infantil e em } 35,5 \% \text { foi alcançada a meta } \\
\text { mínima de investigação. Nos modelos bivariados para avaliação da investigação de pelo menos um óbito, foram } \\
\text { observadas associações estatisticamente significantes com maior porte populacional, maiores valores de Índice } \\
\text { de Desenvolvimento Humano, existência de Comitê de Investigação e de leito obstétrico no município; não foram } \\
\text { observadas associações com a cobertura da Estratégia Saúde da Família e existência de responsável técnico } \\
\text { no município. } \mathrm{Na} \text { análise ajustada, a investigação de pelo menos um óbito infantil esteve associada a porte } \\
\text { populacional }(\mathrm{OR}=4,02) \text { e existência de leito obstétrico }(\mathrm{OR}=2,68) \text {. O alcance da meta municipal mínima esteve } \\
\text { associado apenas com a existência de leito obstétrico no município }(\mathrm{OR}=1,76) \text {. }\end{array}$ \\
\hline $\begin{array}{l}\text { MARQUIEVIZ J, } \\
\text { et al., } 2013\end{array}$ & $\begin{array}{l}\text { Verificou-se um aumento expressivo de } 127,63 \% \text { no número de Equipes de Saúde da Família, com um aumento } \\
\text { da cobertura em } 76,28 \% \text {. Existiu também uma preocupação com relação à capacitação continuada destas } \\
\text { equipes o que repercutiu de forma positiva no aumento de exames para diagnóstico realizado, redução do } \\
\text { número de casos novos, redução da proporção de abandono do tratamento e da taxa de mortalidade relacionada } \\
\text { à tuberculose. }\end{array}$ \\
\hline $\begin{array}{l}\text { GOMES } \\
\text { TGACB, et al., } \\
2016\end{array}$ & $\begin{array}{l}\text { Em 2000, a classificação nacional da taxa de mortalidade na infância era média (30,1/1000 NV), enquanto em } \\
2011 \text {, baixa }(17,7 / 1000 \mathrm{NV}) \text {. }\end{array}$ \\
\hline $\begin{array}{l}\text { PAZÓ RG, et } \\
\text { al., } 2017\end{array}$ & $\begin{array}{l}\text { Houve redução das taxas de ICSAP na medida em que cresceu à taxa de cobertura da Estratégia Saúde da } \\
\text { Família }(0,60 \text {, IC: } 0,56-0,66) \text {, a proporção de médicos }(0,90 \text {, IC: } 0,84-0,96) \text {, de pretos e pardos }(0,88 \text {, IC: } 0,83- \\
0,93) \text { e de pessoas com ensino médio }(0,87 \text {, IC: } 0,76-0,99) \text {. A expansão e consolidação da Estratégia Saúde da } \\
\text { Família são importantes para a redução das ICSAP no estado dentre outros fatores, como recursos de saúde e } \\
\text { fatores socioeconômicos. }\end{array}$ \\
\hline $\begin{array}{l}\text { ZARLOTTI C, et } \\
\text { al., } 2017\end{array}$ & $\begin{array}{l}\text { Houve redução de } 54,4 \% \text { nas ICSAP no período estudado. Observamos uma redução das ICSAP sobre o total } \\
\text { de internações de } 19,9 \% \text {, para } 16,5 \% \text {. A redução na taxa de ICSAP apresentou forte correlação com o aumento } \\
\text { da cobertura do PSF. }\end{array}$ \\
\hline $\begin{array}{l}\text { SILVA-JÚNIOR } \\
\text { VB, et al., } 2018\end{array}$ & $\begin{array}{l}\text { Em relação à taxa de óbitos por DIPs/100 mil habitantes, quando comparado o último com o primeiro ano da } \\
\text { série histórica, observa-se um acréscimo de } 2 \text { óbitos na taxa do país. Em relação à razão média (proporção de } \\
\text { óbitos por DIPs/proporção de internações por DIPs), de } 2011 \text { a } 2015 \text {, é possível perceber que } 57 \% \text { das } \\
\text { internações por esse grupo de doenças geram óbitos. }\end{array}$ \\
\hline
\end{tabular}

Legenda: Abreviações: OR: Odds Ratio; NV: nascidos vivos; ICSAP: internações por condições sensíveis à atenção primária; IC: Intervalo de Confiança; PSF: Programa Saúde da Família; DIPs: doenças infecciosas e parasitárias. Fonte: Ribeiro AF, et al., 2019. 
Em relação aos temas trabalhados nos artigos, houve predominância de discussões sobre impacto da ESF nos indicadores de saúde na atenção básica, internações por condições sensíveis à atenção primária e resultados alcançados com a Estratégia Saúde da Família. Outros temas também se destacam como mortalidade infantil, controle da tuberculose na Estratégia Saúde da Família e doenças infecciosas e parasitárias.

As ações descritas nos artigos foram desenvolvidas pelos profissionais de saúde atuantes nas Unidades Básicas de Saúde (UBS), em sua maioria, agentes comunitários de saúde, enfermeiros, técnicos de enfermagem, médicos e cirurgiões-dentistas, tendo sido excluídos profissionais em licença-maternidade. Muitos artigos foram executados por graduandos e pós-graduandos dos cursos de Educação Física, Enfermagem, Medicina e da área de Saúde Coletiva. No que se diz respeito ao tipo de estudo foram feitos quatro estudos ecológicos, um estudo epidemiológico e dois estudos descritivos.

\section{DISCUSSÃO}

Os indicadores de saúde são medidas-síntese que englobam informações acerca de características e dimensões do estado de saúde, mas também de como o sistema de saúde tem funcionado. Têm um propósito de retratar a situação sanitária de uma população e, de mesmo modo, contribuir para a vigilância de condições de saúde da mesma. Ademais, são ferramentas úteis à gestão, uma vez que facilita o monitoramento de objetivos e metas em saúde e o aprimoramento das equipes, no que se refere à capacidade de análise da situação de saúde, em todos os níveis.

A sua criação se deu com o intuito de quantificar e avaliar tal conjunto de informações obtidas, a partir do sistema público de saúde, no âmbito da ESF. Tal estratégia tem como objetivo reverter a lógica do modelo hospitalocêntrico, priorizando ações de prevenção, promoção e recuperação da saúde. Assim, melhorias nos indicadores de saúde são fundamentais para atestar sua eficiência e associam-se a menores despesas e melhor desempenho macroeconômico (RIPSA, 2008; GUEDES HM, et al., 2007; MACINKO J e MENDONÇA CS, 2018).

O estudo de Guedes HM, et al. (2007), avaliou os resultados de ações de atenção básica antes e depois da implantação da ESF no município de Timóteo/MG, nos anos 1999 e 2005. Os dados levados em conta foram número de visitas domiciliares por profissional; número de procedimentos realizados; número de atendimentos de puericultura/pré-natal e prevenção de câncer cérvico-uterino; número de atendimentos urgência/emergência e internação hospitalar; e indivíduos inscritos nos programas.

Observou-se, por exemplo, aumento das visitas domiciliares, fundamentais para estreitamento da relação profissional/usuário. As visitas médicas cresceram 45,09\% e as de agentes comunitários de saúde (ACSs) $38,91 \%$. Esse salto no número de visitas permite uma promoção de saúde mais efetiva, a partir do contato direto com o usuário inserido em sua realidade distinta, transmitindo mais informações acerca da importância do cuidado e prevenção, além do acompanhamento das condições de saúde dos usuários.

Ainda no mesmo estudo, aliando-se à promoção da saúde realizada de maneira satisfatória ao nível da atenção básica, houve um decréscimo do número de internações hospitalares em 2005 em 84,26\%, comparando-se com o ano de 1999. Essa redução é resultado das ações de controle das doenças, contendo a piora dos quadros clínicos e o risco de complicações. Desse modo, os resultados mostraram uma melhoria na saúde da comunidade analisada, com uma maior atuação da equipe da ESF em prol do cuidado da mesma (GUEDES HM, et al., 2007).

Dentre os indicadores dos artigos analisados, os relacionados à tuberculose destacam-se, já que a enfermidade é considerada um problema de saúde pública e coloca o Brasil entre os 22 países que concentram a maior carga da doença (FREGONA G, 2007; MARQUIEVIZ J, et al., 2013). No estudo de Marquieviz J, et al. (2013), verificou-se queda de 19,21\% na notificação de novos casos de tuberculose na cidade de Curitiba/PR entre os anos de 2000 a 2009, ao mesmo tempo em que houve um aumento no número de culturas para diagnóstico (8.646 em 2005 para 13.569 em 2009) e no número de Equipes de Saúde da Família (de 127,63\%), acompanhadas de uma redução de 55,04\% na taxa de mortalidade pela doença. Tais dados reafirmam a importância da ESF no controle da tuberculose no município. 
Outro parâmetro para avaliar a qualidade da atenção básica diz respeito às internações por condições sensíveis à atenção primária (ICSAP), referindo-se a capacidade resolutiva da mesma (PAZÓ RG, et al., 2017; ZARLOTTI C, et al., 2017). Segundo Pazó RG, et al. (2017), as ICSAP reduziram em $28,79 \%$ no período de 2000 a 2014 nos municípios do estado do Espírito Santo (ES), com destaque para as internações de idosos e adultos, sendo que a cobertura da ESF no ES cresceu em $151 \%$ nesse mesmo período.

Considerando o mesmo indicador, o estudo ecológico de Zarlotti C, et al. (2017), realizado na cidade de Petrópolis/RJ, com análise dos dados do Sistema de Informações Hospitalares do Sistema Único de Saúde (SIH-SUS) entre os anos de 1999-2013, demonstrou redução de 54,4\% de ICSAP e aumento da cobertura populacional da ESF de 2,23\% em 1999 para 40,16\% em 2013. Esses achados fortalecem a associação entre a consolidação e aumento da cobertura da ESF, bem como a qualidade e efetivação de ações, e o controle/diminuição das ICSAP.

Tudo isso associado às melhorias no acompanhamento de condições crônicas, no diagnóstico e acesso a medicamentos, uma vez que autores já apontaram altas taxas de ICSAP, especialmente nas cidades que contavam com menor cobertura de Saúde da Família, o que prejudicava o acesso aos serviços de saúde na atenção básica, por parte da população (PAZÓ RG, et al., 2017; ZARLOTTI C, et al., 2017; PINTO LF e GIOVANELLA L, 2018).

Os estudos de Santana M, et al. (2012) e Gomes TGACB, et al. (2016), seguem uma linha de análise semelhante, ao levarem em conta o papel da atenção básica, sobretudo da ESF, na mortalidade infantil, consequentemente na taxa desse indicador.

Segundo Gomes TGACB, et al. (2016), o destaque é para a região Nordeste do país, com 49,7\% de redução nessa taxa, embora mencionem que o Brasil, de modo geral, vem apresentando uma tendência constante de queda. E no período avaliado de 2000 a 2011 , mostraram que "apenas" $27,5 \%$ da população nordestina não contavam com acesso à atenção básica, apontando ainda aumento da cobertura nacional em $36 \%$.

Por outro lado, Santana M, et al. (2012), os quais tiveram como unidade de análise os 417 municípios do estado da Bahia em 2008, trazem que não se observou associação estatisticamente significativa entre a investigação de pelo menos um óbito infantil com a cobertura municipal da ESF e existência de responsável técnico no município (responsável pela estratégia de Vigilância do Óbito Infantil na Secretaria Municipal de Saúde, implantada em 2005 no referido estado). O estudo ainda indica que 48,9\% dos municípios baianos tiveram registro de pelo menos um óbito infantil no ano considerado, sendo que em 26 deles não houve registro de óbitos de menores de um ano e os percentuais dessa investigação superaram os $100 \%$ em apenas seis municípios.

Outra condição monitorada foram as doenças infecciosas e parasitárias (DIPs), que têm grande potencial de disseminação, configurando-se como um problema de saúde pública, além de se relacionarem com as condições de vida da população. O estudo de Silva-Júnior VB, et al. (2018), analisou internações e óbitos pelas DIPs e a cobertura de ESF no Brasil no período de 2006 a 2015. Quanto à cobertura, observou-se tendência crescente, atingindo $61 \%$ da população no último ano examinado, com ênfase para a região Nordeste, a qual apresentou maior resultado ( $77 \%$ de cobertura), enquanto a região Sudeste atingia metade da população, ficando para trás entre todas as regiões.

Entretanto, a cobertura do serviço não garante o acesso da população ao mesmo, pois o Nordeste apresentou altas taxas de internação. As regiões Sul e Sudeste ficaram abaixo da média nacional quanto à taxa de internação por DIPs no período observado, apresentando bons resultados. Em contrapartida, a última ficou acima da média nacional na taxa de mortalidade por DIPs. Infere-se que as internações, nessas regiões, acontecem com base no potencial das DIPs de causarem óbito, quando já estão em grau evolutivo ou quando são mais letais (SILVA-JÚNIOR VB, et al., 2018).

Mesmo com a adoção do Programa Nacional de Imunizações, criado em 1973, o qual obteve êxito desde a sua criação no controle de várias doenças infecciosas e tendo a ESF como ponto de vacinação principal, algumas DIPs não apresentam medidas de prevenção que sejam eficazes e por estarem relacionadas às condições de vida da população, isso tem dificultado a melhoria das taxas de maneira homogênea entre as 
grandes regiões do país (BRASIL, 2003; CARMO EH, et al., 2003; WALDMAN EA e SATO APS, 2016; SILVAJÚNIOR VB, et al., 2018).

Esta revisão apresentou como limitações o fato de a pesquisa por artigos ter sido feita somente na plataforma da Biblioteca Virtual em Saúde e utilizando apenas duas bases de dados, MEDLINE e LILACS. Além disso, não foram encontrados trabalhos que abordassem os demais indicadores de saúde da atenção básica, os quais não foram discutidos neste estudo. Assim, recomenda-se a busca por artigos em outras fontes de dados.

Entretanto, apesar das barreiras citadas, o presente estudo encontra sua importância pautada por sumarizar e consolidar conhecimentos e dados a respeito do impacto da Estratégia Saúde da Família na Atenção Básica, o que contribui, por exemplo, para o direcionamento de políticas públicas e melhor aplicação dos recursos de saúde.

\section{CONSIDERAÇÕES FINAIS}

Percebeu-se um impacto positivo da ESF sobre os indicadores de saúde analisados nos estudos apresentados, uma vez que houve uma melhoria significativa nos mesmos, acarretando em diferentes vantagens para o serviço de saúde pública nas grandes regiões e nos municípios que foram fonte de análise. Entretanto, ressalta-se que, além da expansão da cobertura e número de equipes de ESF, é necessário que o serviço prestado ofereça acessibilidade adequada e qualidade na assistência, para que as ações operacionalizadas sejam, de fato, efetivas.

\section{REFERÊNCIAS}

1. BRASIL. 8ํㅡㄹ Conferência Nacional de Saúde, de 17 a 21 de março de 1986. Diário Oficial da União, 1986. Disponível em: https://bvsms.saude.gov.br/bvs/publicacoes/8_conferencia_nacional_saude_relatorio_final.pdf. Acesso em 23 nov. 2019.

2. BRASIL. Constituição da República Federativa do Brasil de 1988. Diário Oficial da União, 1988. Disponível em: http://www.planalto.gov.br/ccivil_03/constituicao/constituicao.htm. Acesso em: 23 nov. 2019.

3. BRASIL. Lei $n^{\circ} \times 8.080$, de 19 de setembro de 1990. Dispõe sobre as condições para a promoção, proteção e recuperação da saúde, a organização e o funcionamento dos serviços correspondentes e dá outras providências. Diário Oficial da União, 1990a. Disponível em: http://www.planalto.gov.br/ccivil_03/leis/l8080.htm. Acesso em: 23 nov. 2019.

4. BRASIL. Lei no 8.142, de 28 de dezembro de 1990. Dispõe sobre a participação da comunidade na gestão do Sistema Único de Saúde (SUS) e sobre as transferências intergovernamentais de recursos financeiros na área da saúde e dá outras providências. Diário Oficial da União, 1990b. Disponível em: http://www.planalto.gov.br/ccivil_03/leis/l8142.htm. Acesso em: 23 nov. 2019.

5. BRASIL. Ministério da Saúde. Portaria ํㅜ 1.444, de 28 de dezembro de 2000. Estabelece incentivo financeiro para a reorganização da atenção à saúde bucal prestada nos municípios por meio do Programa de Saúde da Família. Diário $\begin{array}{lllll}\text { Oficial União, } & \text { da } & \text { Disponível } & \end{array}$ http://www1.saude.rs.gov.br/dados/11652497918841\%20Portaria\%20N\%BA\%201444\%20de\%2028\%20dez\%20de \%202000.pdf. Acesso em: 23 nov. 2019.

6. BRASIL. Ministério da Saúde. Programa Nacional de Imunizações. Brasília: Ministério da Saúde, 2003. Disponível em: https://bvsms.saude.gov.br/bvs/publicacoes/livro_30_anos_pni.pdf. Acesso em: 23 nov. 2019.

7. BRASIL. Portaria no 2.436, de 21 de setembro de 2017. Aprova a Política Nacional de Atenção Básica (PNAB), estabelecendo a revisão de diretrizes para a organização da Atenção Básica, no âmbito do Sistema Único de Saúde (SUS). Diário Oficial União, da $2017 . \quad$ Disponível em: https://bvsms.saude.gov.br/bvs/saudelegis/gm/2017/prt2436_22_09_2017.html. Acesso em: 23 nov. 2019.

8. BRASIL. Portaria GAB/MS nำ2.488, de 21 de outubro de 2011. Aprova a Política Nacional de Atenção Básica, estabelecendo a revisão de diretrizes e normas para a organização da Atenção Básica, para a Estratégia Saúde da Família (ESF) e o Programa de Agentes Comunitários de Saúde (PACS). Diário Oficial da União, 2011. Disponível em: http://bvsms.saude.gov.br/bvs/saudelegis/gm/2011/prt2488_21_10_2011.html. Acesso em: 23 nov. 2019.

9. BRASIL. Portaria no 648 , de 28 de março de 2006. Aprova a Política Nacional de Atenção Básica, estabelecendo a revisão de diretrizes e normas para a organização da Atenção Básica para o Programa Saúde da Família (PSF) e o Programa Agentes Comunitários de Saúde (PACS). Diário Oficial da União, 2006. Disponível em: http://bvsms.saude.gov.br/bvs/saudelegis/gm/2006/prt0648_28_03_2006.html. Acesso em: 23 nov. 2019.

10. BRASIL. Portaria no 1.886/GM, de 18 de dezembro de 1997. Aprova as Normas e Diretrizes do Programa de Agentes Comunitários de Saúde e do Programa de Saúde da Família. Diário Oficial da União, 1997. Disponível em: http://189.28.128.100/dab/docs/legislacao/portaria1886_18_12_97.pdf. Acesso em: 23 nov. 2019.

11. CARMO EH, et al. Mudanças nos padrões de morbimortalidade da população brasileira: os desafios para um novo 
século. Epidemiol Serv Saúde, 2003; 12(2):63-75.

12. FREGONA G. Contribuição da Estratégia Saúde da Família para o controle da tuberculose no Espírito Santo [dissertação de Mestrado]. Vitória: Universidade Federal do Espírito Santo, 2007.

13. GOMES TGACB, et al. Mortalidade na infância no Brasil e regiões no período de 2000 a 2011 : o impacto da atenção básica. Comun Ciênc Saúde, 2016; 27(4):259-66.

14. GUEDES HM, et al. Resultados alcançados com a estratégia saúde da família após cinco anos de implantação em uma cidade do interior de Minas Gerais. Rev Min Enferm, 2007; 11(4):363-68.

15. MACINKO J, MENDONÇA CS. Estratégia Saúde da Família, um forte modelo de Atenção Primária à Saúde que traz resultados. Saúde Debate, 2018; 42(Espec. 1):18-37.

16. MARQUIEVIZ J, et al. A Estratégia de Saúde da Família no controle da tuberculose em Curitiba (PR) [tese de Doutorado em Saúde Coletiva]. Curitiba: Curso de Ciência e Saúde Coletiva da Universidade Tecnológica Federal do Paraná, 2013.

17. MOHER D, et al. The PRISMA Group. Preferred Reporting Items for Systematic Reviews and Meta-Analyses: The PRISMA Statement. Traduzido por: Taís Freire Galvão e Thais de Souza Andrade Pansani; retro-traduzido por: David Harrad. Epidemiol Serv Saúde, 2015; 24(2):335-42.

18. PAZÓ RG, et al. Panorama das internações por condições sensíveis à atenção primária no Espírito Santo, Brasil, 2000 a 2014. Rev Bras Med Fam Comunidade, 2017; 12(39):1-12.

19. PINTO LF, GIOVANELLA L. Do Programa à Estratégia de Saúde da Família: expansão de acesso e redução das internações por condições sensíveis à atenção básica (ICSAB). Ciên Saúde Colet, 2018; 23(6): 1903-14.

20. REDE INTERAGENCIAL DE INFORMAÇÃO PARA A SAÚDE (RIPSA). Indicadores básicos para a saúde no Brasil: conceitos e aplicações. Organização Pan-Americana da Saúde, 2008.

21. RONCALLI AG, LIMA KC. Impacto do Programa Saúde da Família sobre indicadores de saúde da criança em municípios de grande porte da região Nordeste do Brasil. Ciên Saúde Colet, 2006; 11(3):713-24.

22. SANTANA M, et al. Efeito da Estratégia Saúde da Família na vigilância de óbitos infantis. Rev Saúde Públ, 2012; 46(1):59-67.

23. SILVA-JÚNIOR VB, et al. Interface entre as doenças infecciosas e parasitárias e a Estratégia Saúde da Família no Brasil [tese de Doutorado em Saúde Coletiva]. Vitória de Santo Antão: Universidade Federal de Pernambuco, 2018.

24. THE JOANNA BRIGGS INSTITUTE (JBI). The Joanna Briggs Institute Reviewers' Manual 2015: methodology for JBI scoping reviews. Adelaide: JBI, 2015.

25. WALDMAN EA, SATO APS. Trajetória das doenças infecciosas no Brasil nos últimos 50 anos: um contínuo desafio. Rev Saúde Públ, 2016; 50(68):50-68.

26. ZARLOTTI C, et al. Internações por condições sensíveis à atenção primária após a implantação da estratégia saúde da família no município de Petrópolis/RJ. J Res Fundam Care Online, 2017; 9(3):811-7. 